\title{
Anaesthesia in a Toxic Environment: Pressurised Intraperitoneal Aerosol Chemotherapy: A Retrospective Analysis
}

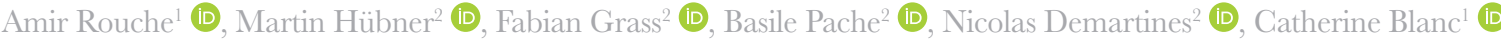 \\ ${ }^{1}$ Department of Anaesthesiology, Lausanne University Hospital (CHUV), Lausanne, Switzerland \\ ${ }^{2}$ Department of Visceral Surgery, Lausanne University Hospital (CHUV), Lausanne, Switzerland
}

Cite this article as: Rouche A, Hübner M, Grass F, Pache B, Demartines N, Blanc C. Anaesthesia in a Toxic Environment: Pressurised Intraperitoneal Aerosol Chemotherapy: A Retrospective Analysis. Turk J Anaesthesiol Reanim 2020; 48(4): 273-9.

\begin{abstract}
Objective: Pressurised intraperitoneal aerosol chemotherapy (PIPAC) is a new type of intraperitoneal chemotherapy for peritoneal carcinosis via minimally invasive surgery. This technique's specificity is the remote application of the therapy because of the potential risk of exposure to toxic products. The present paper summarises the important aspects of PIPAC and analyses the anaesthetic outcomes.

Methods: This retrospective study included all patients undergoing PIPAC treatment between January 2015 and February 2018. Data on protocol adherence and perioperative anaesthetic complications and postoperative nausea and vomiting (PONV) and pain levels (visual analogue scale $0-10)$ from recovery room to $72 \mathrm{~h}$ were analysed.

Results: The overall analysis included 193 PIPAC procedures on 87 patients. Protocol adherence was high as regards the use of propofol $(100 \%)$, rocuronium $(98 \%)$, antiemetic prophylaxis $(99 \%)$ and lidocaine intravenous (i.v.) $(87 \%)$. No accidental exposure to chemotherapy occurred during the study period. Of the 87 patients, $6.3 \%$ suffered delayed recovery, $58 \%$ due to hypothermia and $42 \%$ due to excessive sedation or curarisation. In the recovery room, $16 \%$ of patients suffered moderate to severe pain, requiring $>8 \mathrm{mg}$ of morphine i.v., with average doses of $13.7 \mathrm{mg}$. Median postoperative pain scores were 1 and 3 at $12 \mathrm{~h}$ and 0 and 0 at $72 \mathrm{~h}$ at rest and mobilisation, respectively. PONV was observed in $<10 \%$ of patients during the first $12 \mathrm{~h}$, but in $40 \%$ at $72 \mathrm{~h}$.
\end{abstract}

Conclusion: A dedicated anaesthetic protocol and intraoperative safety checklist facilitates safe, well-tolerated anaesthesia for PIPAC treatments.

Keywords: Adverse effects, doxorubicin, laparoscopy, peritoneal metastases, pressurised intraperitoneal chemotherapy

\section{Introduction}

Peritoneal cancer $(\mathrm{PC})$ is a challenging pathology to diagnose and treat. Without therapeutic management, expected survival time is $<6$ months for PC of colorectal origin or primitive diseases of the peritoneum (1-3). Palliative systemic chemotherapy has a 5 -year survival of $<15 \%$ due to low penetration of the agents and a high incidence of complications. Since 1990, hyperthermic intraperitoneal chemotherapy (HIPEC) in combination with cytoreductive surgery (CRS) has improved outcomes, and the median survival time of patients increased to 22.9 months in a selected cohort of patients (4-6). However, CRS+HIPEG is associated with significant morbidity and mortality rates (42\% and 3.8\%, respectively), even in specialist referral centres, and quality of life $(\mathrm{QoL})$ is impaired for months after the procedure $(4,7,8)$. Therefore, only patients in good general condition can be considered for this treatment. Furthermore, HIPEC has important pharmacokinetic limitations, namely single-dose administration and poor distribution and tissue penetration (9).

Pressurised intraperitoneal aerosol chemotherapy (PIPAC) is a new type of intraperitoneal drug delivery which overcomes some of the above limitations (10). It consists of an intraperitoneal application of vaporised aerosol chemo- 
therapy via minimally invasive surgery using a specific nebuliser $\left(\right.$ CapnoPen $\left.^{\circledR}\right)$. This approach has been shown to improve distribution and tissue penetration considerably (10-12).

Furthermore, PIPAC can be applied repeatedly, thus increasing the potential to achieve local control of recurrent $\mathrm{PC}$ and allowing sequential tumour sampling during laparoscopy. Moreover, chemotherapy doses applied during PIPAC are approximately 10 times smaller than systemic chemotherapy, thus reducing systemic exposure (13).

The present recommendations are for three applications of PIPAC over 3 months. The chemotherapy regimens currently proposed are oxaliplatin alone for colorectal cancer and doxorubicin and cisplatin for PGs of other origins. Platinum agents may induce anaphylactic reactions and can irritate the eyes, skin and airways; they are toxic to the kidneys and bone marrow $(14,15)$. Doxorubicin provokes mucosal inflammation, leukopenia and dilated cardiomyopathy. Both cytotoxic drugs are carcinogenic to humans (class 2A according to the International Agency for Research on Cancer) (16-19).

Owing to the potential leaks of aerosolised cytostatics, a special safety protocol is needed to reduce exposure risks to healthcare staff. The currently proposed protocol involves the remote administration of chemotherapy, with no direct access to the patient for approximately $30 \mathrm{~min}$. This is particularly challenging for an anaesthesiology team as it requires adaptations to standard protocols for comparable procedures regarding invasiveness and duration, such as laparoscopic cholecystectomy.

Therefore, the aim of the present study was to summarise the important aspects of this particular procedure from the anaesthetic point of view and analyse anaesthetic outcomes.

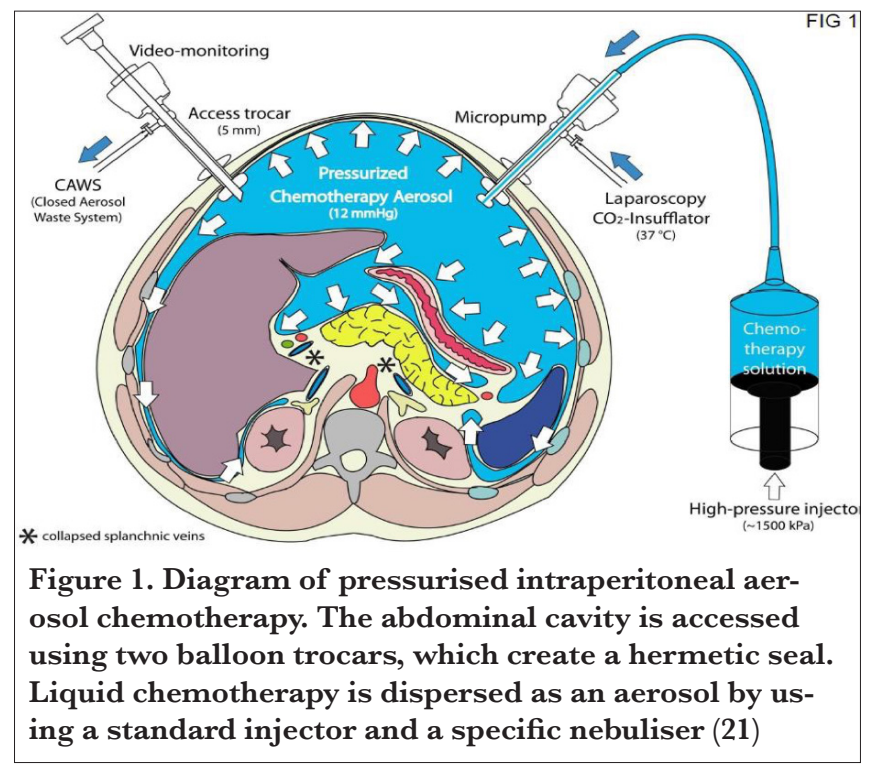

\section{Methods}

This retrospective analysis included all consecutive patients undergoing PIPAC at Lausanne University Hospital between January 2015 and February 2018. All patients were discussed at multidisciplinary tumour boards, and typical indications were isolated peritoneal disease in patients not amenable to CRS+HIPEC.

The study was approved by Lausanne University Hospital's Institutional Review Board (no. 2016-00274) and conducted and reported in compliance with the STROBE criteria and registered online (http://www.researchregistry.com; UIN: 4332). All patients were asked for consent to the use of their anonymised clinical data, via a general consent form, as per Swiss law. Patients refusing to sign the form were excluded from the study.

Anaesthetic protocols and an intraoperative safety checklist and procedure were designed in close collaboration with the surgical team before the study started (20). The anaesthetic protocol and safety checklist are provided as an online Appendix 1 .

\section{Safety considerations}

During the study period, the same surgeon was supported by a dedicated team of anaesthesiologists and instrument technicians for all the PIPAC procedures. Owing to the administration of a carcinogenic chemotherapy, pregnant or breastfeeding women were not allowed to enter the operating room (OR).

Pressurised intraperitoneal aerosol chemotherapy was performed in an OR with a laminar flow, as per safety protocol. Before the vaporisation phase, the surgeon and the entire team run through the safety checklist. During and after the administration phase, healthcare staff must wear a high filtration face mask (FFP3), surgical gown, double nitrile gloves and protective glasses to avoid exposure to the skin and mucosa in case of accidental exposure when entering the OR. With these precautions, entry into the OR due to an anaesthetic problem is allowed if urgently required.

\section{PIPAC procedure}

The procedure lasts approximately $90 \mathrm{~min}$ and is technically comparable to laparoscopic cholecystectomy (Figure 1). Laparoscopy is performed using a standard pressure of $12 \mathrm{mmHg}\left(\mathrm{CO}_{2}\right.$ pneumoperitoneum) using two balloon trocars (21). The first part of the procedure involves an evaluation of the disease extent using the PG index (22). Peritoneal biopsies are obtained, and ascites is removed from the abdominal cavity. Chemotherapy is then vaporised using a high-pressure injector connected to a nebuliser that was de- 
veloped specifically for PIPAC. The injector is remote-controlled, allowing the staff to be outside the OR during the administration of intraperitoneal chemotherapy. The drugs are vaporised for $5 \mathrm{~min}$, and then therapeutic $\mathrm{CO}_{2}$ pneumoperitoneum is maintained for $30 \mathrm{~min}$ to allow drug penetration into the tissues. At the end of the procedure, the pneumoperitoneum is exsufflated via the closed aerosol waste system through two sequential microparticle filters into the anaesthetic air waste system.

\section{Anaesthetic considerations}

PIPAC patients are given general anaesthesia with standard tracheal intubation. Owing to the patient's inaccessibility during the vaporisation phase, monitoring must be possible from outside the OR to allow remote-controlled drug injection if needed.

\section{Positioning and monitoring techniques}

The patient is positioned in the supine position, with legs apart resting on straight pads, allowing the surgeon access between the legs. The left arm is positioned along the body, and the right arm is in abduction at $90^{\circ}$ for venous access. A forced air system (Bair Hugger ${ }^{\mathbf{T M}}$ ) is placed over the upper body. In addition to standard anaesthetic monitoring (electrocardiogram, blood pressure, saturation and temperature), depth of anaesthesia is assessed using a bispectral index, and neuromuscular response is closely monitored using a hand accelerometer. These parameters must be available to the anaesthesiologists outside the OR, either through a window or on a second screen. The patient must be kept strictly and continuously immobile during the vaporisation. Two peripheral venous routes are put in place. The first is used for the induction and maintenance of anaesthesia. The second is connected to a long line and is accessible from outside the OR if re-injection is needed.

Before initiating the vaporisation, monitoring, venous access and patient positioning must be reassessed, and the surgical team goes through the operational safety checklist (online Appendix 2).

\section{Sedation and curarisation}

Propofol is the preferred choice for the induction and maintenance of anaesthesia, as it reduces postoperative nausea and vomiting (PONV) better than volatile anaesthesia. Furthermore, intravenous (i.v.) anaesthesia enables the use of a closed aerosol waste system for exsufflation of the toxic pneumoperitoneum at the end of the procedure.

Curarisation must last until the end of the procedure to ensure complete patient immobility and constant intra-abdominal pressure with a target post-tetanic count of between 5 and 8-10 measured using an automated accelerometer. Ro- curonium is preferred for its ability to be quickly reversed by sugammadex if needed.

\section{Multimodal pain management}

Standard analgesia based on a medium-acting opioid (fentanyl) is given to patients for intubation and surgery. A continuous infusion of lidocaine i.v. is initiated at induction (bolus $\left.1.5 \mathrm{mg} \mathrm{kg}^{-1}\right)$ and continued $\left(2 \mathrm{mg} \mathrm{kg}^{-1} \mathrm{~h}^{-1}\right)$ until departure from the recovery room to reduce opioid use and the side effects of nausea and vomiting and to optimise postoperative analgesia. In addition, patients receive a magnesium sulphate infusion at a rate of $40 \mathrm{mg} \mathrm{kg}^{-1}$ over $10 \mathrm{~min}$.

Postoperative analgesia is based on paracetamol, nonsteroidal anti-inflammatory drugs and morphine on demand for the postoperative period.

\section{Prevention of PONV}

The combination of general anaesthesia and chemotherapy can potentially amplify PONV. Therefore, patients receive an association of three antiemetic drugs: droperidol and dexamethasone at the beginning of the procedure and ondansetron before waking up and for the postoperative period. In cases of intense PONV, fosaprepitant was selected as a rescue treatment.

\section{Data management}

Demographic, surgical and anaesthetic details for all patients were prospectively entered in a computerised, coded database designed specifically for the quality control of the PIPAC cohort. Demographic data included age, sex, body mass index, American Society of Anesthesiologists and cancer origins. Anaesthetic protocol adherence was analysed with regard to the presence of the dedicated anaesthetic team and the protocol's proposed drug usage. Recorded anaesthetic complications included severe arrhythmia, haemodynamic instability, anaphylaxis, mild hypothermia (defined as a temperature from $32.2^{\circ} \mathrm{C}$ to $35.5^{\circ} \mathrm{C}$ ), delayed recovery $(>15$ min after the end of surgery), difficult i.v. access (defined as needing more than two attempts) and moderate to severe pain (defined as needing $>8 \mathrm{mg}$ of morphine in the recovery room). For the first 74 PIPAC procedures, patients described their pain using a visual analogue scale, and the incidence of PONV was assessed from the recovery room until $72 \mathrm{~h}$ and entered in the quality control database.

\section{Statistical analysis}

Statistical analysis was performed using Stata Software (v. 14.2; StataCorp, College Station, TX, USA). Continuous variables were presented as mean \pm standard deviation or median value with range or interquartile range (IQR), as appropriate, depending on the normality of the distribution. 


\section{Results}

A median of 2 (1-7) PIPAC treatments was performed on our 87 patients, with a total of 193 procedures. The cohort's demographic information is shown in Table 1 . The median postoperative length of stay was 2 (IQR 2-3) days.

All surgeries were performed by the same surgeon, whereas anaesthesiologists and nurse anaesthetists from the dedicated team were present at $79 \%$ and $55 \%$ of procedures, respectively. The overall protocol adherence was high with respect to the use of propofol $(100 \%)$, rocuronium $(98 \%)$ and multimodal antiemetic prophylaxis $(99 \%)$. Lidocaine perfusion was used in 169 (87\%) surgi-

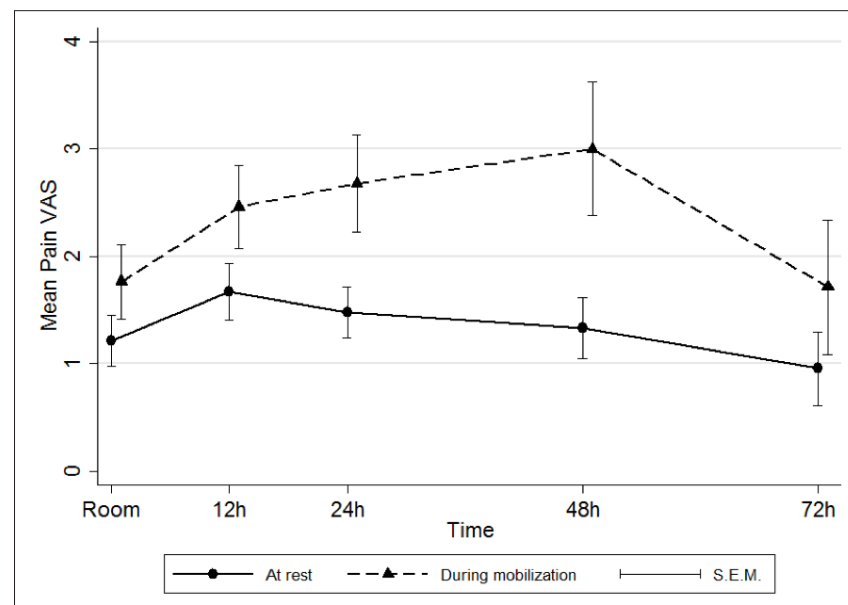

Figure 2. Pain scores at rest and during mobilisation. Evolution of pain scores over time after a PIPAC procedure, at rest and during mobilisation at different time points postoperatively

VAS: visual analogue scale; S.E.M.: standard error of the mean; Room: recovery room

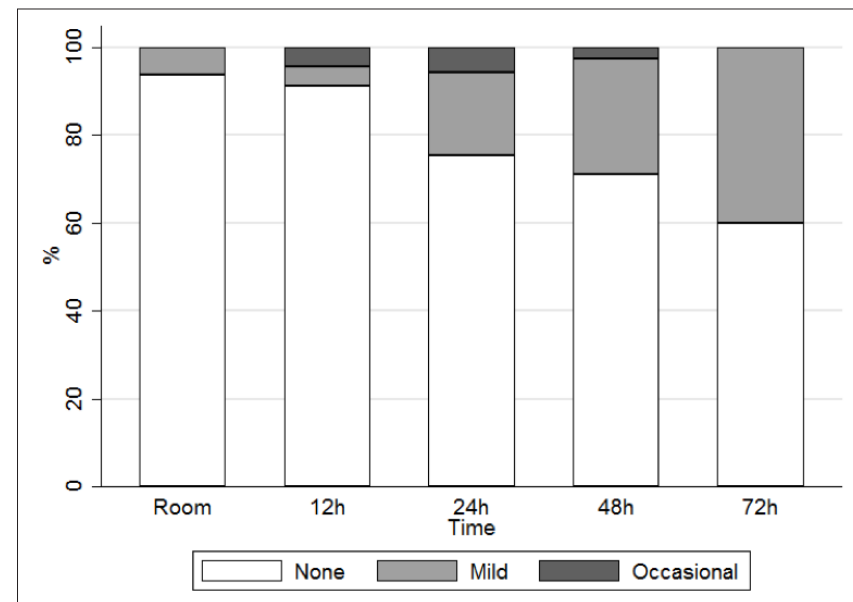

Figure 3. Postoperative nausea and vomiting (PONV). PONV after PIPAC at different time points Room: recovery room; PIPAC: pressurised intraperitoneal aerosol chemotherapy cal procedures and continued until departure from the recovery room. Sugammadex had to be used in $52(27 \%)$ procedures.

Anaesthetic complications occurred in 32 (16.5\%) procedures. Twelve $(6.3 \%)$ patients suffered delayed recovery due to hypothermia $(n=7)$ or excessive sedation or curarisation $(n=5)$. Ten $(5.2 \%)$ patients had mild hypothermia at the end of the procedure. Other anaesthetic complications were 3 difficult intubations, 4 difficult i.v. accesses and 10 mild haemodynamic instabilities during the laparoscopic phase, requiring low-dose vasopressor treatment.

In the recovery room, moderate to severe pain was documented after $31(16 \%)$ procedures, requiring $>8 \mathrm{mg}$ of morphine i.v. and with median doses of $13.7 \mathrm{mg}$ (IQR 8.2-19.2). Median postoperative pain scores were 1 (IQR $0-4)$ and 3 (IQR $0-4)$ at $12 \mathrm{~h}$ and $0(\mathrm{IQR} 0-2)$ and 0 (IQR $0-3)$ at $72 \mathrm{~h}$ at rest and mobilisation, respectively (Figure 2). Symptoms of PONV were present in $6.1 \%$ of patients in the recovery room and in $40 \%$ of the 25 patients who were still in the hospital at 72 h (Figure 3).

No accidental exposure to chemotherapy occurred during the study period. Entry to the OR during the vaporisation phase was necessary $<10$ times, principally for anaesthetic pump dysfunction.

\begin{tabular}{|l|c|}
\hline \multicolumn{2}{|l|}{ Table 1. Patient demographics } \\
\hline No. of patients & $63(55-70)$ \\
Median age, years (IQR) & \\
Sex & $25(27.8 \%)$ \\
Male & $65(72.2 \%)$ \\
Female & $23.7(21.2-26.8)$ \\
Median BMI, kg m-2 (IQR) & \\
ASA & $57(65.5 \%)$ \\
II & $30(34.4 \%)$ \\
III & \\
Aetiology of cancer & $39(44.8 \%)$ \\
Ovarian & $29(33.3 \%)$ \\
Colorectal & $8(9.2 \%)$ \\
Gastric & $4(4.6 \%)$ \\
Mesothelioma & $7(8.1 \%)$ \\
Other & $2(1-7)$ \\
Median no. of PIPAC procedures (range) & $2(2-3,1-20)$ \\
Median length of stay, days (IQR, range) & \\
\hline IQR: interquartile range; BMI: body mass index; ASA: American \\
Society of Anesthesiologists; PIPAC: pressurised intraperitoneal aerosol \\
chemotherapy
\end{tabular}




\section{Discussion}

To the best of our knowledge, this is the first study to analyse the anaesthetic management and complications of patients undergoing PIPAC treatment. This surgical technique is special because the safety drug protocol requires the remote administration of intraperitoneal chemotherapy, limiting access to the patient. By using a dedicated anaesthetic protocol, anaesthesia for PIPAC ensured the safety of patients and healthcare staff, and anaesthetic outcomes were similar to those of comparable procedures.

Pressurised intraperitoneal aerosol chemotherapy is a very new type of treatment, and evidence is so far limited. A first, nine-centre, international survey involved 832 procedures on 349 patients and evaluated the different aspects of PIPAC treatment with respect to surgical methodology. The study demonstrated that the procedures were performed the same way, probably explained by the standardised procedure and the limited number of expert centres (23). A systematic review on PIPAC, published in 2017 by Grass et al. (24), demonstrated that the procedure was safe and well tolerated. Histological response rates for therapy-resistant carcinomatosis of ovarian, colorectal and gastric origins were promising. Furthermore, our group's evaluation of QoL after PIPAC therapy demonstrated no negative impact of the treatment (25).

It has been shown that the safe implementation of PIPAC was possible from the surgical point of view-even with no learning curve-when the existing recommendations and protocols were followed (20). The requirement to be outside the OR and to apply anaesthesia far from the patient is a challenging scenario, but one which has been described previously, for example, in paediatrics with sedation for multiple sessions of radiotherapy (26-28). For the PIPAC procedure, anaesthesia must be given in a potentially toxic environment involving cytotoxic drugs. Although no emanations of cytotoxic agents were detected during experimental procedures and no air contamination was detectable at the surgeon's and anaesthesiologist's workstations (29), strict protocols, a safety checklist and a dedicated team are mandatory for the safe execution of this procedure, including following recommendations on the manipulation of cytotoxic drugs, as for HIPEC procedures (30).

Adherence to the anaesthetic protocol described above was high, despite the staff turnover inherent in a teaching institution. However, a senior anaesthesiologist and referent nurse anaesthetist were available on site, in the event of a problem, and the anaesthetic protocol and safety checklist were available on the department's intranet. This underscores the importance of homogenous pathways in maintaining standardisation regardless of staffing changes.
The overall severity of surgical complications was mild and has already been described by our group. We recorded no micro-pump injector disconnections or accidental vaporisation into the OR air during our series.

By using the recommended protocol, not one major anaesthetic complication was observed in 193 procedures. Hypothermia occurred mainly at the beginning of the study due to the initial technical problems with the laparoscopic-gas heater and/or late positioning of the forced air system and late prevention of hypothermia during anaesthetic induction and installation. However, peripheral venous access was challenging as most patients had few accessible veins due to multiple lines of upfront systemic chemotherapy. Other minor anaesthetic problems, such as haemodynamic instability, difficult intubation and delayed recovery due to excess sedation or curarisation, are inherent to all anaesthetic procedures in a teaching hospital and are not related to PIPAC procedures in particular.

Lidocaine i.v. was added to optimise pain management, but this occurred after the start of our study programme, thus explaining the $87 \%$ adherence rate. Pain scores were low thanks to a multimodal analgesic approach based on paracetamol and nonsteroidal anti-inflammatory drugs, and they were similar to those patients undergoing laparoscopic cholecystectomy (31). Only $26(13.6 \%)$ procedures resulted in patients requiring morphine i.v. in the recovery room. With regard to the $40 \%$ of patients presenting with PONV at $72 \mathrm{~h}$, only complicated and very frail patients had a hospital length of stay that long, thus explaining this high incidence of PONV.

\section{Conclusion}

The anaesthetic management of patients undergoing PIPAC procedures can be performed safely by following the recommendations within a standardised pathway. Subtle adaptations of standard protocols are necessary to adjust to the requirements of the safety protocol, which includes remote administration. Despite this, the anaesthetic management and clinical outcomes of this procedure were comparable with operations of similar invasiveness and duration, such as laparoscopic cholecystectomy.

Ethics Committee Approval: Ethics committee approval was received for this study from the ethics committee of Lausanne University Hospital's Institutional Review Board - Pr. Patrick Francioli (2016-00274).

Informed Consent: Written informed consent was obtained from patients who participated in this study.

Peer-review: Externally peer-reviewed. 
Author Contributions: Concept - M.H.; Design - A.R., M.H., C.B.; Supervision - A.R., M.H., F.G., N.D., G.B.; Resources - N.D., C.B.; Materials - A.R., B.P.; Data Collection and/or Processing A.R., B.P., F.G.; Analysis and/or Interpretation - A.R., B.P., F.G.; Literature Search - A.R.; Writing Manuscript - A.R.; Critical Review - A.R., M.H., F.G., B.P., N.D., G.B.

Conflict of Interest: The authors have no conflicts of interest to declare.

Financial Disclosure: The authors declared that this study has received no financial support.

\section{References}

1. Lemmens VE, Klaver YL, Verwaal VJ, Rutten HJ, Coebergh JW, de Hingh IH. Predictors and survival of synchronous peritoneal carcinomatosis of colorectal origin: a population-based study. Int J Cancer 2011; 128: 2717-25. [CrossRef]

2. Klaver YL, Lemmens VE, Creemers GJ, Rutten HJ, Nienhuijs SW, de Hingh IH. Population-based survival of patients with peritoneal carcinomatosis from colorectal origin in the era of increasing use of palliative chemotherapy. Ann Oncol 2011; 22: 2250-6. [CrossRef]

3. Quere P, Facy O, Manfredi S, Jooste V, Faivre J, Lepage C, et al. Epidemiology, Management, and Survival of Peritoneal Carcinomatosis from Colorectal Cancer: A Population-Based Study. Dis Colon Rectum 2015; 58: 743-52. [GrossRef]

4. Newton AD, Bartlett EK, Karakousis GC. Cytoreductive surgery and hyperthermic intraperitoneal chemotherapy: a review of factors contributing to morbidity and mortality. J Gastrointest Oncol 2016; 7: 99-111.

5. Sugarbaker PH. Cytoreductive surgery and hyperthermic intraperitoneal chemotherapy in the management of gastrointestinal cancers with peritoneal metastases: Progress toward a new standard of care. Cancer Treat Rev 2016; 48: 42-9. [GrossRef]

6. Glehen O, Gilly FN, Boutitie F, Bereder JM, Quenet F, Sideris $\mathrm{L}$, et al. Toward curative treatment of peritoneal carcinomatosis from nonovarian origin by cytoreductive surgery combined with perioperative intraperitoneal chemotherapy: a multi-institutional study of 1,290 patients. Cancer 2010; 116: 5608-18. [CrossRef]

7. Levine EA, Stewart JHt, Shen P, Russell GB, Loggie BL, Votanopoulos KI. Intraperitoneal chemotherapy for peritoneal surface malignancy: experience with 1,000 patients. J Am Coll Surg 2014; 218: 573-85. [CrossRef]

8. Odendahl K, Solass W, Demtröder C, Giger-Pabst U, Zieren J, Tempfer C, et al. Quality of life of patients with end-stage peritoneal metastasis treated with Pressurized IntraPeritoneal Aerosol Chemotherapy (PIPAC). Eur J Surg Oncol 2015; 41: 1379-85. [GrossRef]

9. Dedrick RL, Flessner MF. Pharmacokinetic problems in peritoneal drug administration: tissue penetration and surface exposure. J Natl Cancer Inst 1997; 89: 480-7. [GrossRef]

10. Solaß W, Hetzel A, Nadiradze G, Sagynaliev E, Reymond MA. Description of a novel approach for intraperitoneal drug de- livery and the related device. Surg Endosc 2012; 26: 1849-55. [CrossRef]

11. Reymond M, Solass W. Pressurized Intraperitoneal Aerosol Chemotherapy-Cancer Under Pressure. 1. ed: Walter de Gruyter GmbH \& Co KG, Berlin; 2014. [CrossRef]

12. Khosrawipour V, Khosrawipour T, Kern AJ, Osma A, Kabakci B, Diaz-Carballo D, et al. Distribution pattern and penetration depth of doxorubicin after pressurized intraperitoneal aerosol chemotherapy (PIPAC) in a postmortem swine model. J Cancer Res Clin Oncol 2016; 142: 2275-80. [GrossRef]

13. Solass W, Kerb R, Murdter T, Giger-Pabst U, Strumberg D, Tempfer G, et al. Intraperitoneal chemotherapy of peritoneal carcinomatosis using pressurized aerosol as an alternative to liquid solution: first evidence for efficacy. Ann Surg Oncol 2014; 21: 553-9. [GrossRef]

14. Extra JM, Marty M, Brienza S, Misset JL. Pharmacokinetics and safety profile of oxaliplatin. Semin Oncol 1998; 25: 13-22.

15. Teixeira Farinha H, Grass F, Labgaa I, Pache B, Demartines N, Hübner M. Inflammatory Response and Toxicity After Pressurized IntraPeritoneal Aerosol Chemotherapy. J Cancer 2018; 9: 13-20. [GrossRef]

16. Reymond M, Solass W. PIPAC-Pressurized intraperitoneal aerosol chemotherapy-Cancer under pressure. Walter de Gruyter; 2014. [GrossRef]

17. Demtröder C, Solass W, Zieren J, Strumberg D, Giger-Pabst U, Reymond MA. Pressurized intraperitoneal aerosol chemotherapy with oxaliplatin in colorectal peritoneal metastasis. Colorectal Dis 2016; 18: 364-71. [CrossRef]

18. Nadiradze G, Giger-Pabst U, Zieren J, Strumberg D, Solass W, Reymond MA. Pressurized Intraperitoneal Aerosol Chemotherapy (PIPAC) with Low-Dose Cisplatin and Doxorubicin in Gastric Peritoneal Metastasis. J Gastrointest Surg 2016; 20: 367-73. [GrossRef]

19. IARC. IARC Monographs on the Evaluation of Carcinogenic Risks to Humans. 2006.

20. Hübner M, Teixeira Farinha H, Grass F, Wolfer A, Mathevet P, Hahnloser D, et al. Feasibility and Safety of Pressurized Intraperitoneal Aerosol Chemotherapy for Peritoneal Carcinomatosis: A Retrospective Cohort Study. Gastroenterol Res Pract 2017; 2017: 6852749. [CrossRef]

21. Hübner M, Teixeira H, Boussaha T, Cachemaille M, Lehmann K, Demartines N. PIPAC--Pressurized intraperitoneal aerosol chemotherapy. A novel treatment for peritoneal carcinomatosis. Rev Med Suisse 2015; 11: 1325-30.

22. Mazzei MA, Khader L, Cirigliano A, Cioffi Squitieri N, Guerrini S, Forzoni B, et al. Accuracy of MDCT in the preoperative definition of Peritoneal Cancer Index (PCI) in patients with advanced ovarian cancer who underwent peritonectomy and hyperthermic intraperitoneal chemotherapy (HIPEC). Abdom Imaging 2013; 38: 1422-30. [GrossRef]

23. Nowacki M, Alyami M, Villeneuve L, Mercier F, Hubner M, Willaert W, et al. Multicenter comprehensive methodological and technical analysis of 832 pressurized intraperitoneal aerosol chemotherapy (PIPAC) interventions performed in 349 patients for peritoneal carcinomatosis treatment: An international survey study. Eur J Surg Oncol 2018; 44: 991-6. [CrossRef]

24. Grass F, Vuagniaux A, Teixeira-Farinha H, Lehmann K, Demartines N, Hübner M. Systematic review of pressurized 
intraperitoneal aerosol chemotherapy for the treatment of advanced peritoneal carcinomatosis. Br J Surg 2017; 104: 669-78. [CrossRef]

25. Teixeira Farinha H, Grass F, Kefleyesus A, Achtari C, Romain B, Montemurro M, et al. Impact of Pressurized Intraperitoneal Aerosol Chemotherapy on Quality of Life and Symptoms in Patients with Peritoneal Carcinomatosis: A Retrospective Cohort Study. Gastroenterol Res Pract 2017; 2017: 4596176. [CrossRef]

26. Anghelescu DL, Burgoyne LL, Liu W, Hankins GM, Cheng C, Beckham PA, et al. Safe anesthesia for radiotherapy in pediatric oncology: St. Jude Children's Research Hospital Experience, 2004-2006. Int J Radiat Oncol Biol Phys 2008; 71: 491-7. [CrossRef]

27. McFadyen JG, Pelly N, Orr RJ. Sedation and anesthesia for the pediatric patient undergoing radiation therapy. Curr Opin Anaesthesiol 2011; 24: 433-8. [CrossRef]
28. Gozal D, Gozal Y. Pediatric sedation/anesthesia outside the operating room. Curr Opin Anaesthesiol 2008; 21: 494-8. [CrossRef]

29. Solass W, Giger-Pabst U, Zieren J, Reymond MA. Pressurized intraperitoneal aerosol chemotherapy (PIPAC): occupational health and safety aspects. Ann Surg Oncol 2013; 20: 3504-11. [CrossRef]

30. Kyriazanos I, Kalles V, Stefanopoulos A, Spiliotis J, Mohamed F. Operating personnel safety during the administration of $\mathrm{Hy}-$ perthermic Intraperitoneal Chemotherapy (HIPEC). Surg Oncol 2016; 25: 308-14. [GrossRef]

31. Grass F, Cachemaille M, Blanc C, Fournier N, Halkic N, Demartines N, et al. Is standardized care feasible in the emergency setting? A case matched analysis of patients undergoing laparoscopic cholecystectomy. BMC Surg 2016; 16: 78. [CrossRef] 